MATHEMATICS OF COMPUTATION

Volume 69, Number 229, Pages 269-282

S 0025-5718(99)01078-9

Article electronically published on March 4, 1999

\title{
ON THE ERROR TERM OF SYMMETRIC GAUSS-LOBATTO QUADRATURE FORMULAE FOR ANALYTIC FUNCTIONS
}

\author{
DAVID HUNTER AND GENO NIKOLOV
}

\begin{abstract}
Gauss-Lobatto quadrature formulae associated with symmetric weight functions are considered. The kernel of the remainder term for classes of analytic functions is investigated on elliptical contours. Sufficient conditions are found ensuring that the kernel attains its maximal absolute value at the intersection point of the contour with either the real or the imaginary axis. The results obtained here are an analogue of some recent results of T. Schira concerning Gaussian quadratures.
\end{abstract}

\section{Introduction AND STATEMENT OF RESUlts}

Let $w$ be a nonnegative weight function on the interval $[-1,1]$. We study interpolatory quadrature formulae of the form

$$
Q_{n}[f]:=\sum_{\nu=1}^{n} w_{\nu, n} f\left(x_{\nu, n}\right), \quad-1 \leq x_{1, n}<x_{2, n} \cdots<x_{n, n} \leq 1,
$$

which serve as estimates for the integral

$$
I[f]:=\int_{-1}^{1} w(x) f(x) d x
$$

If the integrand $f$ is analytic in some simply connected and bounded region $G$ of the complex plane containing $[-1,1]$ in its interior, then the remainder term $R_{n}:=I-Q_{n}$ can be expressed as a contour integral over $\Gamma:=\partial G$,

$$
R_{n}[f]=\frac{1}{2 \pi i} \int_{\Gamma} K_{n}(z ; w) f(z) d z
$$

where $K_{n}(\cdot ; w)$ is referred to as the kernel of the functional $R_{n}$ (or of the quadrature formula $Q_{n}$ ), and is given by

$$
K_{n}(z ; w):=\frac{q_{n}(z)}{p_{n}(z)}
$$

Received by the editor October 14, 1997 and, in revised form, March 26, 1998.

1991 Mathematics Subject Classification. Primary 41A55; Secondary 65D30, 65D35.

Key words and phrases. Gauss-Lobatto quadrature, remainder term for analytic functions, contour integral representation, kernel function.

The second author did this work while he was on leave from the Department of Mathematics, University of Sofia, blvd J. Bourchier 5, 1164 Sofia, Bulgaria. He was supported by a grant from the Royal Society, and by the Bulgarian Ministry of Science, Education and Technologies under Grant MM-513/95. 
Here, $p_{n}(z):=c\left(z-x_{1, n}\right) \cdots\left(z-x_{n, n}\right)$ is the nodal polynomial, and for $z \in \mathbb{C} \backslash[-1,1]$ $q_{n}$ is given by

$$
q_{n}(z):=\int_{-1}^{1} w(x) \frac{p_{n}(x)}{z-x} d x
$$

An alternative representation for $K_{n}(z ; w)$ is

$$
K_{n}(z ; w):=R_{n}\left[\frac{1}{z-\cdot}\right]=\int_{-1}^{1} \frac{w(x)}{z-x} d x-\sum_{\nu=1}^{n} \frac{w_{\nu, n}}{z-x_{\nu, n}} .
$$

Formula (1.3) leads to the error estimate

$$
\left|R_{n}[f]\right| \leq \frac{l(\Gamma)}{2 \pi} \max _{z \in \Gamma}\left|K_{n}(z ; w)\right| \max _{z \in \Gamma}|f(z)|
$$

(for a different approach to the estimation of $R_{n}[f]$, see [6]).

Typical choices of the contour $\Gamma$ are concentric circles centred at the origin or confocal ellipses $\mathcal{E}_{\varrho}$ with foci at \pm 1 and sum of semiaxes $\varrho>1$,

$$
\mathcal{E}_{\varrho}:=\left\{z \in \mathbb{C}: z=\frac{1}{2}\left(\varrho e^{i \theta}+\varrho^{-1} e^{-i \theta}\right), 0 \leq \theta<2 \pi\right\}, \quad \varrho>1 .
$$

The derivation of adequate bounds for $\left|R_{n}[f]\right|$ on the basis of (1.7) is possible only if good estimates for $\max _{z \in \Gamma}\left|K_{n}(z ; w)\right|$ are available. Especially useful is knowledge of the location of the extremal point $\xi \in \Gamma$, at which $\left|K_{n}\right|$ attains its maximum. In such a case, instead of looking for upper bounds for $\max _{z \in \Gamma}\left|K_{n}(z ; w)\right|$ one can simply try to calculate $\left|K_{n}(\xi ; w)\right|$. In general, this may not be an easy task, but in the case where $Q_{n}$ is a Gauss-type quadrature formula there exist effective algorithms for calculation of $K_{n}(z ; w)$ at any point $z$ outside [-1,1] (see Gautschi and Varga [4]).

The problem of finding the maximum point of the kernel $\left|K_{n}(z ; w)\right|$ of Gausstype quadrature formulae on circular or elliptic contours has received considerable attention (see [1], [2], [3], [4], [5], [7]). Typically, the results in this direction are obtained either for special weights (e.g., for Chebyshev weights) or under restriction of monotonicity type (e.g., $w(x) / w(-x)$ is monotone). Under such assumptions, usually it is shown that the maximum point $\xi$ is an intersection point of the contour with either the real or the imaginary axis, or is located near one of these points.

Let $w$ be a symmetric weight function on $[-1,1]$, i.e., $w(-x)=w(x)$ for every $x \in[-1,1]$. We shall investigate symmetric generalized Gauss-Lobatto quadrature formulae associated with $w$,

$$
Q_{n}^{(\sigma)}[f]:=\sum_{\mu=0}^{\sigma-1} a_{\mu, n}^{(\sigma)}\left[f^{(\mu)}(-1)+(-1)^{\mu} f^{(\mu)}(1)\right]+\sum_{\nu=1}^{n} w_{\nu, n}^{(\sigma)} f\left(x_{\nu, n}^{(\sigma)}\right) .
$$

The formulae $Q_{n}^{(\sigma)}$ are uniquely defined by the property that their remainder term $R_{n}^{(\sigma)}:=I-Q_{n}^{(\sigma)}$ vanishes identically on the set of all algebraic polynomials of degree at most $2(n+\sigma)-1$. In particular, $Q_{n}^{(0)}$ is the $n$-point Gauss quadrature formula

$$
Q_{n}^{\mathrm{G}}[f]:=\sum_{\nu=1}^{n} w_{\nu, n}^{\mathrm{G}} f\left(x_{\nu, n}^{\mathrm{G}}\right),
$$


$Q_{n}^{(1)}$ is the $(n+2)$-point Gauss-Lobatto quadrature formula

$$
Q_{n+2}^{\mathrm{Lo}}[f]:=a_{n}^{\mathrm{Lo}}[f(-1)+f(1)]+\sum_{\nu=1}^{n} w_{\nu, n}^{\mathrm{Lo}} f\left(x_{\nu, n}^{\mathrm{Lo}}\right),
$$

and $Q_{n}^{(2)}$ is the $(n+2)$-point Gauss-Lobatto quadrature formula with double end nodes $Q_{n+2}^{\mathrm{Lo}, d}$ (each of these three formulae is associated with the weight function $w)$.

The kernel $K_{n}^{\mathrm{G}}(\cdot ; w)$ of the Gauss quadrature formula (1.10) has been investigated on elliptical contours in a recent paper of T. Schira [10].

Denote by $z_{r}=z_{r}(\varrho)$ and $z_{i}=z_{i}(\varrho)$ the intersection points of the ellipse $\mathcal{E}_{\varrho}$ with the positive branches of the real and the imaginary axis, respectively; i.e.,

$$
z_{r}:=\frac{\varrho+\varrho^{-1}}{2}, \quad z_{i}:=\frac{i\left(\varrho-\varrho^{-1}\right)}{2} .
$$

For weight functions $w(x)$ symmetric in $(-1,1)$ and satisfying the condition that either $w(x) \sqrt{1-x^{2}}$ is increasing or $w(x) / \sqrt{1-x^{2}}$ is decreasing on $(0,1)$, Schira proved that the extremum point $\xi$ coincides with $z_{r}$ in the first case, and with $z_{i}$ in the second case, provided $\varrho$ is not less than some given value. The assumptions for $w$ are satisfied; e.g., for the Gegenbauer weight function

$$
w_{\alpha}(x):=\left(1-x^{2}\right)^{\alpha}, \quad \alpha>-1, \alpha \notin(-1 / 2,1 / 2) .
$$

Schira has proved a result of a similar nature for the Gauss-Lobatto quadrature formula (1.11).

Theorem A ([8, Satz 4.4]). Let $K_{n+2}^{\mathrm{Lo}}(\cdot ; w)$ be the kernel of the Gauss-Lobatto quadrature formula (1.11) associated with a symmetric weight function $w$ on $(-1,1)$. If $w(x) \sqrt{1-x^{2}}$ is increasing on $(0,1)$, then

$$
\max _{x \in \mathcal{E}_{\varrho}}\left|K_{n+2}^{\mathrm{Lo}}(z ; w)\right|=\left|K_{n+2}^{\mathrm{Lo}}\left(z_{r} ; w\right)\right| \quad \text { for all } \varrho \geq \varrho_{*}:=\frac{\sqrt{2}}{2}(1+\sqrt{3})
$$

( for $n=0$ this result holds for all $\varrho>1$ ).

In this paper we apply Schira's approach to obtain an analogue of Theorem A for symmetric weight functions $w$ such that $w(x) / \sqrt{1-x^{2}}$ is decreasing on $(0,1)$ (Theorem 1.2). Partially, this approach turns out to be applicable also to the generalized symmetric Gauss-Lobatto quadrature formulae (1.9) (Theorem 1.3).

For any nonnegative integer $\sigma$, denote by $\pi_{m}^{(\sigma)}$ the $m$ th orthonormal polynomial with respect to the weight function

$$
w^{(\sigma)}(x):=\left(1-x^{2}\right)^{\sigma} w(x),
$$

and by $c_{m}^{(\sigma)}$ its leading coefficient (without loss of generality we assume $c_{m}^{(\sigma)}>0$ ). The basic ingredient of the proof of Theorems 1.2 and 1.3 is the following expansion formula for the kernel $K_{n}^{(\sigma)}(\cdot ; w)$ of the generalized Gauss-Lobatto quadrature formulae (1.9).

Theorem 1.1. The kernel $K_{n}^{(\sigma)}(\cdot ; w)$ of the generalized Gauss-Lobatto quadrature formulae (1.9) associated with a symmetric weight function $w$ on $(-1,1)$ has the 
representation

$K_{n}^{(\sigma)}(z ; w)=(-1)^{\sigma} \sum_{\nu=0}^{\infty} \frac{c_{n+2 \nu+2}^{(\sigma)}}{c_{n+2 \nu}^{(\sigma)}} \frac{z}{\left(z^{2}-1\right)^{\sigma} \pi_{n+2 \nu}^{(\sigma)}(z) \pi_{n+2 \nu+2}^{(\sigma)}(z)} \quad(z \in \mathbb{C} \backslash[-1,1])$.

We note that the special cases $\sigma=0$ and $\sigma=1$ of Theorem 1.1 were already proved by Schira (see [10, Theorem 3.1] and $[8,(4.13)]$ ).

A useful feature of the formula (1.12) is that it allows us to replace the examination of $K_{n}^{(\sigma)}(\cdot ; w)$ by the examination of the terms appearing in its expansion. If all these terms attain their maximal moduli at $z_{r}$ (or at $z_{i}$ ), then this property is inherited by the kernel. With the help of Theorem 1.1 we prove the following theorems.

Theorem 1.2. Let $K_{n+2}^{\mathrm{Lo}}(\cdot ; w)$ be the kernel of the Gauss-Lobatto quadrature formula (1.11) associated with a symmetric weight function $w$ on $(-1,1)$. If $w(x) / \sqrt{1-x^{2}}$ is decreasing on $(0,1)$, then for $n \geq 3$

$$
\max _{z \in \mathcal{E}_{\varrho}}\left|K_{n+2}^{\mathrm{Lo}}(z ; w)\right|=\left|K_{n+2}^{\mathrm{Lo}}\left(z_{i} ; w\right)\right| \quad \text { for all } \varrho \geq \varrho_{*},
$$

where

$$
\varrho_{*}= \begin{cases}3.88 & \text { if } n \text { is odd } \\ 3.72 & \text { if } n \text { is even }\end{cases}
$$

Theorem 1.3. Let $w$ be a symmetric weight function such that $w(x) \sqrt{1-x^{2}}$ is increasing on $(0,1)$. Then for the kernel $K_{n+2}^{\mathrm{Lo}, d}(\cdot ; w)$ of the Gauss-Lobatto quadrature formula with double end nodes we have

$$
\max _{z \in \mathcal{E}_{\varrho}}\left|K_{n+2}^{\mathrm{Lo}, d}(z ; w)\right|=\left|K_{n, 2}^{\mathrm{Lo}, d}\left(z_{r} ; w\right)\right| \quad \text { for all } \varrho \geq \varrho_{*}:=\frac{\sqrt{2}}{2}(1+\sqrt{3}) .
$$

\section{Auxiliary Results}

The following two lemmas are taken from Schira's work [10].

Lemma 2.1 ([10, Lemma 2.1]). Let $w$ and $\tilde{w}$ be two symmetric weight functions on $(-1,1)$ and let the zeros $x_{k, n}$ and $\tilde{x}_{k, n}$ of the corresponding $n$ th-degree orthogonal polynomials be arranged in decreasing order. If $w / \tilde{w}$ is increasing on $(0,1)$, then the inequalities $x_{k, n} \geq \tilde{x}_{k, n}$ hold for $k=1, \ldots,\left[\frac{n}{2}\right]$.

Lemma 2.2 ([10, Lemma 4.1]). The function

$$
g(z):=\frac{z^{2}-s^{2}}{z^{2}-t^{2}}
$$

with $s, t \in(0,1)$ has the property that on every ellipse $\mathcal{E}_{\varrho}$ with $\varrho \geq \varrho_{*}:=\frac{\sqrt{2}}{2}(1+\sqrt{3})$ we have

$$
\max _{z \in \mathcal{E}_{\varrho}}|g(z)|= \begin{cases}g\left(z_{r}\right) & \text { if } s<t \\ g\left(z_{i}\right) & \text { if } s>t\end{cases}
$$


The conformal map $z=\left(u+u^{-1}\right) / 2$ transforms concentric circles $|u|=\varrho(\varrho>1)$ into confocal ellipses $\mathcal{E}_{\varrho}$. The Chebyshev polynomials of the first and the second kind are expressed as

$$
T_{n}(z)=\frac{u^{n}+u^{-n}}{2}
$$

and

$$
U_{n}(z)=\frac{u^{n+1}-u^{-n-1}}{u-u^{-1}},
$$

respectively. We shall use the familiar notation (see [2], [3], [4])

$$
a_{m}(\varrho):=\frac{1}{2}\left(\varrho^{m}+\varrho^{-m}\right) .
$$

The following simple lemma describes a relation between the quantities $a_{m}(\varrho)$.

Lemma 2.3. For every fixed $\varrho_{0}>1$ and for every nonnegative integer $m$ the following inequality holds true:

$$
a_{2 m}(\varrho) \leq d_{m} a_{2 m+2}(\varrho) \quad \text { for every } \varrho \geq \varrho_{0},
$$

where

$$
d_{m}=\frac{\varrho_{0}^{4 m}+1}{\varrho_{0}^{4 m+2}} .
$$

Proof. We examine first for which positive constants $c$ the inequality

$$
a_{2 m+2}(\varrho) \geq c \varrho^{2} a_{2 m}(\varrho)
$$

is fulfilled for every $\varrho \geq \varrho_{0}$. Using the representation (2.3), we conclude that (2.6) will hold if

$$
c \leq \frac{\varrho^{4 m}}{\varrho^{4 m}+1} .
$$

Since the right-hand side of this last inequality increases as $\varrho$ increases, the choice

$$
c=\frac{\varrho_{0}^{4 m}}{\varrho_{0}^{4 m}+1}
$$

guarantees the validity of $(2.6)$ for all $\varrho \geq \varrho_{0}$. Thus, for $\varrho \geq \varrho_{0}$ we have

$$
a_{2 m}(\varrho) \leq \frac{\varrho_{0}^{4 m}+1}{\varrho^{2} \varrho_{0}^{4 m}} a_{2 m+2}(\varrho) \leq \frac{\varrho_{0}^{4 m}+1}{\varrho_{0}^{4 m+2}} a_{2 m+2}(\varrho) .
$$

The lemma is proved.

We shall also need the simple inequalities given by the next lemma.

Lemma 2.4. For every integer $m$ and for every $\theta \in \mathbb{R}$

$$
\begin{gathered}
\frac{\sin ^{2} 2 m \theta}{\cos ^{2} \theta} \leq 4 m^{2} ; \\
\frac{\cos ^{2}(2 m+1) \theta}{\cos ^{2} \theta} \leq(2 m+1)^{2} .
\end{gathered}
$$

Let

$$
v_{m}(z):=\frac{U_{m-2}^{\prime}(z)}{U_{m}^{\prime}(z)} .
$$

The following lemma reveals a property of the function $v_{m}(z)$, which may be of independent interest, and which can be made more precise, if necessary. 
Lemma 2.5. For any integer $m \geq 6$ and for every $\varrho \geq 2.3$ we have

$$
\max _{z \in \mathcal{E}_{\varrho}}\left|v_{m}(z)\right|=\left|v_{m}\left(z_{i}\right)\right| .
$$

Proof. Using the differential equation

$$
\left(1-z^{2}\right) T_{n}^{\prime \prime}(z)-z T_{n}^{\prime}(z)+n^{2} T_{n}(z)=0
$$

and the identity $T_{n+1}^{\prime}=(n+1) U_{n}$, we obtain

$$
v_{m}(z)=\frac{(m-1) T_{m-1}(z)-z U_{m-2}(z)}{(m+1) T_{m+1}(z)-z U_{m}(z)} .
$$

For $z \in \mathcal{E}_{\varrho}$ formulae (2.1) and (2.2) yield

$$
\left|v_{m}(z)\right|^{2}=\frac{g_{m-2}(\varrho, \theta)}{g_{m}(\varrho, \theta)},
$$

where

$$
\begin{aligned}
g_{n}(\varrho, \theta)= & n^{2}\left[a_{2 n+4}(\varrho)-\cos (2 n+4) \theta\right]-2 n(n+2) \cos 2 \theta a_{2 n+2}(\varrho) \\
& +(n+2)^{2}\left[a_{2 n}(\varrho)-\cos 2 n \theta\right]+2 n(n+2) \cos (2 n+2) \theta a_{2}(\varrho) .
\end{aligned}
$$

The cases of odd and even $m$ require separate consideration, but, as the idea of the proof is the same, we restrict ourselves to studying only the case of $m$ even. In this case, we have

$$
g_{m-2}(\varrho, \theta)=A_{m-2}(\varrho)-2 B_{m-2}(\varrho, \theta),
$$

where

$$
\begin{aligned}
A_{m-2}(\varrho)= & (m-2)^{2} a_{2 m}(\varrho)+2(m-2) m a_{2 m-2}(\varrho) \\
& +m^{2} a_{2 m-4}(\varrho)-2(m-2) m a_{2}(\varrho)-m^{2}-(m-2)^{2}, \\
B_{m-2}(\varrho, \theta)=2 & (m-2) m \cos ^{2} \theta a_{2 m-2}(\varrho)-2(m-2) m \cos ^{2}(m-1) \theta a_{2}(\varrho) \\
- & (m-2)^{2} \sin ^{2} m \theta-m^{2} \sin ^{2}(m-2) \theta,
\end{aligned}
$$

and analogous relations hold for $A_{m}(\varrho)$ and $B_{m}(\varrho, \theta)$. We conclude on the basis of equations (2.7) and (2.9) that

$$
\left|v_{m}\left(z_{i}\right)\right|^{2}-\left|v_{m}(z)\right|^{2}=\frac{A_{m-2}(\varrho)}{A_{m}(\varrho)}-\frac{A_{m-2}(\varrho)-2 B_{m-2}(\varrho, \theta)}{A_{m}(\varrho)-2 B_{m}(\varrho, \theta)},
$$

and consequently

$$
\operatorname{sign}\left\{\left|v_{m}\left(z_{i}\right)\right|^{2}-\left|v_{m}(z)\right|^{2}\right\}=\operatorname{sign}\left\{A_{m}(\varrho) B_{m-2}(\varrho, \theta)-A_{m-2}(\varrho) B_{m}(\varrho, \theta)\right\} .
$$

Thus, we need to examine the sign of the last expression. After some straightforward (though rather tedious) calculations, in the process of which we repeatedly use the identity $a_{k} a_{l}=\left(a_{k+l}+a_{|k-l|}\right) / 2$, we arrive at the representation

$$
A_{m} B_{m-2}-A_{m-2} B_{m}=\sum_{k=0}^{2 m+1} c_{k}(\theta) a_{2 k}(\varrho),
$$

where

$$
\begin{gathered}
c_{2 m+1}(\theta)=4(m-2) m \cos ^{2} \theta, \\
c_{2 m-1}(\theta)=-4(m+2) m \cos ^{2} \theta, \\
c_{m+3}(\theta)=-m^{3}(m-2) \cos ^{2}(m-1) \theta,
\end{gathered}
$$




$$
\begin{aligned}
& c_{m+2}(\theta)=2 m^{2}\left(m^{2}-4\right)\left[\cos ^{2} \theta-\cos ^{2}(m-1) \theta\right] \\
& -m^{2}\left[(m-2)^{2} \sin ^{2} m \theta+m^{2} \sin ^{2}(m-2) \theta\right], \\
& c_{m+1}(\theta)=2 m(m+2)\left[m^{2}+(m-2)^{2}\right] \cos ^{2} \theta+m(m-2)^{2}(m+2) \cos ^{2}(m+1) \theta \\
& -(m-2) m\left[m^{2}+(m+2)^{2}\right] \cos ^{2}(m-1) \theta \\
& -2 m(m+2)\left[(m-2)^{2} \sin ^{2} m \theta+m^{2} \sin ^{2}(m-2) \theta\right], \\
& c_{m}(\theta)=2 m^{2}\left(m^{2}-4\right)\left[\cos ^{2}(m+1) \theta-\cos ^{2}(m-1) \theta\right] \\
& +m^{2}\left[(m-2)^{2} \sin ^{2}(m+2) \theta-(m+2)^{2} \sin ^{2}(m-2) \theta\right], \\
& c_{m-1}(\theta)=m(m+2)\left[m^{2}+(m-2)^{2}\right] \cos ^{2}(m+1) \theta \\
& +2(m-2) m\left[m^{2} \sin ^{2}(m+2) \theta+(m+2)^{2} \sin ^{2} m \theta\right] \\
& -2(m-2) m\left[(m+2)^{2}+m^{2}\right] \cos ^{2} \theta \\
& -m(m-2)(m+2)^{2} \cos ^{2}(m-1) \theta \text {, } \\
& c_{m-2}(\theta)=2 m^{2}\left(m^{2}-4\right)\left[\cos ^{2}(m+1) \theta-\cos ^{2} \theta\right] \\
& +m^{2}\left[m^{2} \sin ^{2}(m+2) \theta+(m+2)^{2} \sin ^{2} m \theta\right], \\
& c_{m-3}(\theta)=m^{3}(m+2) \cos ^{2}(m+1) \theta, \\
& c_{3}(\theta)=-4 m^{3} \cos ^{2} \theta, \\
& c_{2}(\theta)=2 m^{2}\left(m^{2}-4\right)\left[\cos ^{2}(m-1) \theta-\cos ^{2}(m+1) \theta\right], \\
& c_{1}(\theta)=4 m\left(m^{2}-4\right) \cos ^{2} \theta+2(m-2) m\left[(m+2)^{2}+m^{2}\right] \cos ^{2}(m-1) \theta \\
& +2(m+2) m\left[(m-2)^{2} \sin ^{2} m \theta+m^{2} \sin ^{2}(m-2) \theta\right] \\
& -2(m-2) m\left[m^{2} \sin ^{2}(m+2) \theta+(m+2)^{2} \sin ^{2} m \theta\right] \\
& -2(m+2) m\left[m^{2}+(m-2)^{2}\right] \cos ^{2}(m+1) \theta, \\
& c_{0}(\theta)=2 m^{2}\left(m^{2}-4\right)\left[\cos ^{2}(m-1) \theta-\cos ^{2}(m+1) \theta\right] \\
& +\left[(m+2)^{2}+m^{2}\right]\left[(m-2)^{2} \sin ^{2} m \theta+m^{2} \sin ^{2}(m-2) \theta\right] \\
& -\left[m^{2}+(m-2)^{2}\right]\left[m^{2} \sin ^{2}(m+2) \theta+(m+2)^{2} \sin ^{2} m \theta\right],
\end{aligned}
$$

and all the remaining coefficients are equal to zero.

Further, we divide $A_{m} B_{m-2}-A_{m-2} B_{m}$ by $\cos ^{2} \theta$ and apply Lemma 2.4 to obtain the estimate

(2.10)

$$
\begin{aligned}
\frac{A_{m} B_{m-2}-A_{m-2} B_{m}}{\cos ^{2} \theta} \geq & 4 m(m-2) a_{4 m+2}-4 m(m+2) a_{4 m-2} \\
& -m^{3}(m-1)^{2}(m-2) a_{2 m+6}-4 m^{3}(m-2)^{2}(m+1) a_{2 m+4} \\
& -6 m^{6} a_{2 m+2}-3 m^{6} a_{2 m}-m^{6} a_{2 m-2}-m^{6} a_{2 m-4} \\
& -m^{6} a_{6}-3 m^{6} a_{4}-11 m^{6} a_{2}-10 m^{6} a_{0} \\
= & : 4 m(m-2) a_{4 m+2}-4 m(m+2) a_{4 m-2}-\sum_{j=0}^{m+3} \alpha_{j} a_{2 j} .
\end{aligned}
$$


Note that, for the sake of simplicity, the estimates for the coefficients of the "lower degree" terms, (i.e., the $a_{j}$ 's with indices less than $2 m+4$ ) are rather crude. The reason why we may be content with such a rough estimation is that the quantities $a_{m}(\varrho)$ increase rapidly as $m$ increases, if $\varrho$ is not too close to 1 , and as a result only the first few terms in the right-hand side of (2.10) are of importance.

The right-hand side of (2.10) is now estimated with the help of Lemma 2.3. For $\varrho \geq 2.3$ the inequality $a_{2 j}(\varrho) \leq d_{j} a_{2 j+2}(\varrho)$ holds, e.g., with $d_{0}=0.4, d_{1}=0.2$ and with $d_{m}=0.19=: d$ for all $m \geq 2$. Thus, we obtain successively

$$
\begin{gathered}
10 a_{0} \leq 4 a_{2}, \quad 10 a_{0}+11 a_{2} \leq 3 a_{4}, \quad 10 a_{0}+11 a_{2}+3 a_{4} \leq 1.14 a_{6}, \\
10 a_{0}+11 a_{2}+3 a_{4}+a_{6} \leq 0.41 a_{8} \leq 0.41 a_{2 m-4},
\end{gathered}
$$

and repeating this procedure we finally get

$$
\begin{aligned}
\sum_{j=0}^{m+3} \alpha_{j} a_{2 j} & \leq\left(2 m^{6}-6.28 m^{5}+5 m^{4}+1.04 m^{3}\right) a_{2 m+6} \\
& \leq 2 m^{5}(m-2) a_{2 m+6} \leq 2 m^{5}(m-2) d^{m-2} a_{4 m+2} .
\end{aligned}
$$

We also have, for $m \geq 6$,

$$
\begin{aligned}
4 m(m+2) a_{4 m-2} & \leq 4 m(m-2) \frac{m+2}{m-2} d^{2} a_{4 m+2} \\
& \leq 0.29 m(m-2) a_{4 m+2} .
\end{aligned}
$$

Combining (2.11) and (2.12), we obtain

$$
\begin{gathered}
4 m(m-2) a_{4 m+2}-4 m(m+2) a_{4 m-2}-\sum_{j=0}^{m+3} \alpha_{j} a_{2 j} \\
\geq\left[3.71-2 m^{4} d^{m-2}\right] m(m-2) a_{4 m+2} .
\end{gathered}
$$

Therefore, to prove Lemma 2.5 for $m$ even, it suffices to show that the quantity $\delta(m):=2 m^{4} d^{m-2}$ does not exceed 3.71 for every $m \geq 6$. The latter is easily seen, for $\delta(m)$ decreases monotonically as $m$ increases and $\delta(6) \leq 3.38$.

Repetition of the above reasoning in the case of $m$ odd leads to the same conclusion. This completes the proof of Lemma 2.5.

For any natural $m \geq 2$ set

$$
\mu_{m}(z):=\frac{z}{\left(z^{2}-1\right) U_{m}^{\prime}(z) U_{m+2}^{\prime}(z)}, \quad \nu_{m}(z):=\frac{z}{\left(z^{2}-1\right)^{2} U_{m}^{\prime}(z) U_{m+2}^{\prime}(z)} .
$$

Lemma 2.6. For every natural number $m \geq 2$ and for every $\varrho \geq \varrho_{*}=\frac{\sqrt{2}}{2}(1+\sqrt{3})$ we have

$$
\max _{z \in \mathcal{E}_{\varrho}}\left|\nu_{m}(z)\right|=\nu_{m}\left(z_{r}\right) .
$$

Proof. The case $m=2$ is verified directly, so we suppose that $m \geq 3$. We write

$$
\nu_{m}(z)=z \xi_{m}(z) \xi_{m+2}(z)
$$

where

$$
\xi_{m}(z)=\frac{1}{\left(z^{2}-1\right) U_{m}^{\prime}(z)}
$$


Clearly, the lemma will be proved if we succeed in showing that for every $m \geq 3$ and for every $\varrho \geq \varrho_{*}$

$$
\max _{z \in \mathcal{E}_{\varrho}}\left|\xi_{m}(z)\right|=\xi_{m}\left(z_{r}\right) .
$$

Indeed, having established (2.14), we will obtain

$$
\begin{aligned}
\max _{z \in \mathcal{E}_{e}}\left|\nu_{m}(z)\right| & \leq \max _{z \in \mathcal{E}_{\varrho}}|z| \max _{z \in \mathcal{E}_{\varrho}}\left|\xi_{m}(z)\right| \max _{z \in \mathcal{E}_{\varrho}}\left|\xi_{m+2}(z)\right| \\
& =z_{r} \xi_{m}\left(z_{r}\right) \xi_{m+2}\left(z_{r}\right)=\nu_{m}\left(z_{r}\right) .
\end{aligned}
$$

Using well-known properties of Chebyshev polynomials, we get

$$
\left|\xi_{m}(z)\right|^{2}=\frac{4\left(a_{2}(\varrho)-\cos 2 \theta\right)}{g_{m}(\varrho, \theta)},
$$

where $g_{m}$ is defined as in (2.8). We write

$$
g_{m}(\varrho, \theta)=g_{m}(\varrho, 0)+\left(g_{m}(\varrho, \theta)-g_{m}(\varrho, 0)\right)=C_{m}(\varrho)+2 D_{m}(\varrho, \theta),
$$

where

$$
\begin{aligned}
C_{m}= & m^{2} a_{2 m+4}-2 m(m+2) a_{2 m+2}+(m+2)^{2} a_{2 m} \\
& +2 m(m+2) a_{2}-m^{2}-(m+2)^{2}
\end{aligned}
$$

and

$$
\begin{aligned}
D_{m}= & 2 m(m+2)\left[a_{2 m+2} \sin ^{2} \theta-a_{2} \sin ^{2}(m+1) \theta\right] \\
& +m^{2} \sin ^{2}(m+2) \theta+(m+2)^{2} \sin ^{2} m \theta .
\end{aligned}
$$

Thus

$$
\left|\xi_{m}\left(z_{r}\right)\right|^{2}-\left|\xi_{m}(z)\right|^{2}=\frac{4\left(a_{2}(\varrho)-1\right)}{C_{m}(\varrho)}-\frac{4\left(a_{2}(\varrho)-1+2 \sin ^{2} \theta\right)}{C_{m}(\varrho)+2 D_{m}(\varrho, \theta)},
$$

and consequently

$$
\begin{aligned}
\operatorname{sign}\left\{\left|\xi_{m}\left(z_{r}\right)\right|^{2}-\left|\xi_{m}(z)\right|^{2}\right\} & =\operatorname{sign}\left\{\left(a_{2}(\varrho)-1\right) D_{m}(\varrho, \theta)-C_{m}(\varrho) \sin ^{2} \theta\right\} \\
& =: \operatorname{sign} \varepsilon(\varrho, \theta) .
\end{aligned}
$$

The calculation of $\varepsilon(\varrho, \theta)$ yields

$$
\begin{aligned}
\varepsilon(\varrho, \theta)= & 2 m \sin ^{2} \theta a_{2 m+4}-2(m+2) \sin ^{2} \theta a_{2 m}-m(m+2) \sin ^{2}(m+1) \theta a_{4} \\
& +[m \sin (m+2) \theta+(m+2) \sin m \theta]^{2}\left(a_{2}-1\right) \\
& +m(m+2) \sin ^{2}(m+1) \theta+4 \sin ^{2} \theta .
\end{aligned}
$$

We ignore the last nonnegative terms and apply Lemma 2.4 to obtain

$$
\sin ^{-2} \theta \varepsilon(\varrho, \theta) \geq 2 m a_{2 m+4}-2(m+2) a_{2 m}-m(m+2)(m+1)^{2} a_{4} .
$$

Now we prove that for $\varrho \geq \varrho_{*}$ the right-hand side of (2.15) is nonnegative. The reasoning is the same as in the final part of the proof of Lemma 2.5. According to Lemma 2.3, for $\varrho \geq \varrho_{*}$ and for every $j \geq 2$ we have $a_{2 j} \leq d a_{2 j+2}$, where we may choose $d=0.27$. For $\varrho \geq \varrho_{*}$ we obtain

$$
a_{4} \leq(0.27)^{m} a_{2 m+4}
$$

and

$$
2(m+2) a_{2 m}=2 m \frac{m+2}{m} a_{2 m} \leq 2 m \frac{5}{3}(0.27)^{2} a_{2 m+4}<0.243 m a_{2 m+4} .
$$


Hence

$$
\begin{gathered}
2 m a_{2 m+4}-2(m+2) a_{2 m}-m(m+2)(m+1)^{2} a_{4} \\
\geq\left[1.757-(m+1)^{2}(m+2)(0.27)^{m}\right] m a_{2 m+4} .
\end{gathered}
$$

The term in the square brackets is positive for $m \geq 3$, and this completes the proof of Lemma 2.6.

Next we examine the functions $\mu_{m}(z)$.

Lemma 2.7. For every natural $m \geq 4$ and for every $\varrho \geq \varrho_{*}$ we have

$$
\max _{z \in \mathcal{E}_{\varrho}}\left|\mu_{m}(z)\right|=\left|\mu_{m}\left(z_{i}\right)\right|
$$

where

$$
\varrho_{*}= \begin{cases}3.88 & \text { if } m \text { is even, } \\ 3.72 & \text { if } m \text { is odd } .\end{cases}
$$

Proof. Using the explicit form of $\mu_{4}$ and $\mu_{5}$ and applying Lemmas 2.3 and 2.5, we obtain after some lengthy but straightforward calculations that

$$
\max _{z \in \mathcal{E}_{\varrho}}\left|\mu_{4}(z)\right|=\left|\mu_{4}\left(z_{i}\right)\right| \quad \text { for every } \varrho \geq 3.88
$$

and

$$
\max _{z \in \mathcal{E}_{e}}\left|\mu_{5}(z)\right|=\left|\mu_{5}\left(z_{i}\right)\right| \quad \text { for every } \varrho \geq 3.72 .
$$

The proof then proceeds by induction. Assuming that (2.16) is true for some natural $m-2 \geq 4$, we apply Lemma 2.5 to conclude that for $z \in \mathcal{E}_{\varrho}$ and $\varrho \geq \varrho_{*}$

$$
\begin{aligned}
\left|\mu_{m}(z)\right| & =\left|\frac{U_{m-2}^{\prime}(z)}{U_{m}^{\prime}(z)}\right|\left|\frac{U_{m}^{\prime}(z)}{U_{m+2}^{\prime}(z)}\right|\left|\mu_{m-2}(z)\right| \\
& \leq\left|\frac{U_{m-2}^{\prime}\left(z_{i}\right)}{U_{m}^{\prime}\left(z_{i}\right)}\right|\left|\frac{U_{m}^{\prime}\left(z_{i}\right)}{U_{m+2}^{\prime}\left(z_{i}\right)}\right|\left|\mu_{m-2}\left(z_{i}\right)\right| \\
& =\left|\mu_{m}\left(z_{i}\right)\right| .
\end{aligned}
$$

The lemma is proved.

\section{Proof of Results}

Proof of Theorem 1.1. It is well known that the nodes $\left\{x_{\nu, n}^{(\sigma)}\right\}_{\nu=1}^{n}$ of the generalized Gauss-Lobatto quadrature formulae (1.9) are exactly the zeros of $\pi_{n}^{(\sigma)}$, the $n$th orthonormal polynomial with respect to the weight function $w^{(\sigma)}(x)=\left(1-x^{2}\right)^{\sigma} w(x)$. The functions $p_{n}=p_{n}^{(\sigma)}$ and $q_{n}=q_{n}^{(\sigma)}$ in this case are given by

$$
p_{n}^{(\sigma)}(x)=\left(1-x^{2}\right)^{\sigma} \pi_{n}^{(\sigma)}(x)
$$

and

$$
q_{n}^{(\sigma)}(z)=\int_{-1}^{1} w_{\sigma}(x) \frac{\pi_{n}^{(\sigma)}(x)}{z-x} d x
$$

We observe that

$$
K_{n}^{(\sigma)}(z ; w)=\frac{q_{n}^{(\sigma)}(z)}{p_{n}^{(\sigma)}(z)}=\frac{K_{n}^{\mathrm{G}}\left(z ; w^{(\sigma)}\right)}{\left(1-z^{2}\right)^{\sigma}}
$$


where $K_{n}^{\mathrm{G}}\left(\cdot ; w^{(\sigma)}\right)$ is the kernel of the $n$-point Gauss quadrature formula associated with the weight function $w^{(\sigma)}(x)$. To complete the proof of Theorem 1.1 we only have to repeat Schira's arguments in the proof of ([10, Theorem 3.1]).

In the special case $\sigma=1$ (i.e., when $Q_{n}^{(\sigma)}$ is the customary Gauss-Lobatto quadrature formula $Q_{n+2}^{\mathrm{Lo}}$ ) Theorem 1.1 yields

$$
\begin{aligned}
K_{n+2}^{\mathrm{Lo}}(z ; w) & =-\sum_{j=0}^{\infty} \frac{c_{n+2 j+2}}{c_{n+2 j}} \frac{z}{\left(z^{2}-1\right) \pi_{n+2 j}(z) \pi_{n+2 j+2}(z)} \\
& =:-\sum_{j=0}^{\infty} \frac{c_{n+2 j+2}}{c_{n+2 j}} \psi_{n+2 j}(z),
\end{aligned}
$$

where $\pi_{m}$ is the $m$ th orthogonal polynomial associated with the weight $\left(1-x^{2}\right) w(x)$, and $c_{m}>0$ is its leading coefficient. In particular, for the second Chebyshev weight function $w_{1 / 2}(x)=\sqrt{1-x^{2}}$ we have

$$
\begin{aligned}
K_{n+2}^{\mathrm{Lo}}\left(z ; w_{1 / 2}\right) & =-\sum_{j=0}^{\infty} \gamma_{j} \frac{z}{\left(z^{2}-1\right) U_{n+2 j+1}^{\prime}(z) U_{n+2 j+3}^{\prime}(z)} \\
& =:-\sum_{j=0}^{\infty} \gamma_{j} \mu_{n+2 j+1}(z)
\end{aligned}
$$

with positive constants $\gamma_{j}$.

For the kernel $K_{n+2}^{\mathrm{Lo}, d}\left(\cdot ; w_{-1 / 2}\right)$ of the Gauss-Lobatto quadrature formula with double end nodes associated with the first Chebyshev weight function, Theorem 1.1 yields

$$
\begin{aligned}
K_{n+2}^{\mathrm{Lo}, d}\left(z ; w_{-1 / 2}\right) & =\sum_{j=0}^{\infty} \delta_{j} \frac{z}{\left(z^{2}-1\right)^{2} U_{n+2 j+1}^{\prime}(z) U_{n+2 j+3}^{\prime}(z)} \\
& =: \sum_{j=0}^{\infty} \delta_{j} \nu_{n+2 j+1}(z)
\end{aligned}
$$

with positive constants $\delta_{j}$.

Proof of Theorem 1.2. The functions $\psi_{m}(z)$ appearing in the expansion (3.1) can be expressed as

$$
\psi_{m}(z)=\mu_{m+1}(z) \frac{U_{m+1}^{\prime}(z) U_{m+3}^{\prime}(z)}{\pi_{m}(z) \pi_{m+2}(z)} .
$$

Now we compare the terms in series (3.1) and (3.2). The polynomials $U_{m}^{\prime}$ and $\pi_{m}$ are orthogonal with respect to the weight functions $\tilde{w}(x)=\left(1-x^{2}\right)^{3 / 2}$ and $w^{(1)}(x)=\left(1-x^{2}\right) w(x)$, respectively. It is assumed that $w^{(1)}(x) / \tilde{w}(x)$ is monotone decreasing in $(0,1)$, and therefore $\tilde{w}(x) / w^{(1)}(x)$ is monotone increasing therein. Hence we infer from Lemma 2.1 that

$$
\begin{aligned}
\eta_{m}(z):= & \frac{U_{m+1}^{\prime}(z) U_{m+3}^{\prime}(z)}{\pi_{m}(z) \pi_{m+2}(z)}=\prod_{j=1}^{m+1} \frac{z^{2}-s_{j}^{2}}{z^{2}-t_{j}^{2}} \\
& \text { with } 0 \leq t_{j} \leq s_{j}<1(j=1, \ldots, m+1) .
\end{aligned}
$$


The application of Lemma 2.2 to each multiplier in $\eta_{m}$ then yields

$$
\max _{z \in \mathcal{E}_{\varrho}}\left|\eta_{m}(z)\right|=\left|\eta_{m}\left(z_{i}\right)\right| \quad \text { for every } \varrho \geq \frac{\sqrt{2}}{2}(1+\sqrt{3}) .
$$

According to Lemma 2.7, for $m \geq 3$ we have

$$
\max _{z \in \mathcal{E}_{\varrho}}\left|\mu_{m+1}(z)\right|=\left|\mu_{m+1}\left(z_{i}\right)\right|
$$

for every $\varrho \geq 3.88$ if $m$ is odd, and for every $\varrho \geq 3.72$ if $m$ is even. Combining the last two conclusions, we obtain that for $m \geq 3$

$$
\max _{z \in \mathcal{E}_{\varrho}}\left|\psi_{m}(z)\right|=\left|\psi_{m}\left(z_{i}\right)\right| \quad \text { for every } \varrho \geq \varrho_{*},
$$

with $\varrho_{*}$ as defined in Theorem 1.2. Moreover, the observation that the zeros of $\pi_{m}$ are located symmetrically with respect to the origin implies

$$
\left|\psi_{n+2 \nu}\left(z_{i}\right)\right|=i(-1)^{n} \psi_{n+2 \nu}\left(z_{i}\right) .
$$

Therefore, for $\varrho \geq \varrho_{*}$ the expansion formula (3.1) yields

$$
\begin{aligned}
& \left|K_{n+2}^{\mathrm{Lo}}(z ; w)\right| \leq \sum_{\nu=0}^{\infty} \frac{c_{n+2 \nu+2}}{c_{n+2 \nu}}\left|\psi_{n+2 \nu}(z)\right| \\
& \quad \leq \sum_{\nu=0}^{\infty} \frac{c_{n+2 \nu+2}}{c_{n+2 \nu}}\left|\psi_{n+2 \nu}\left(z_{i}\right)\right|=\left|K_{n+2}^{\mathrm{Lo}}\left(z_{i} ; w\right)\right| .
\end{aligned}
$$

This completes the proof of Theorem 1.2.

Proof of Theorem 1.3. The proof is essentially the same as that of the preceding theorem. In this case we compare pairwise the terms in the series (1.12) (with $\sigma=2$ ) and (3.3), using the relation

$$
\psi_{m}(z)=\nu_{m+1}(z) \frac{U_{m+1}^{\prime}(z) U_{m+3}^{\prime}(z)}{\pi_{m}(z) \pi_{m+2}(z)} .
$$

The claim then follows immediately from Lemmas 2.1, 2.2 and 2.6.

\section{Concluding Remarks}

1. The most important application of Theorem A and Theorems 1.2 and 1.3 is in the case of the Gegenbauer weight function $w_{\alpha}(x)=\left(1-x^{2}\right)^{\alpha}, \alpha>-1$, $\alpha \notin(-1 / 2,1 / 2)$. In this case $w_{\alpha}(x) \sqrt{1-x^{2}}=\left(1-x^{2}\right)^{\alpha+1 / 2}$ and $w_{\alpha}(x) / \sqrt{1-x^{2}}=$ $\left(1-x^{2}\right)^{\alpha-1 / 2}$; hence we immediately obtain the following two corollaries.

Corollary 4.1. The kernel $K_{n+2}^{\mathrm{Lo}}\left(\cdot ; w_{\alpha}\right)$ of the $(n+2)$-point Gauss-Lobatto quadrature formula with respect to the Gegenbauer weight function $w_{\alpha}(x)=\left(1-x^{2}\right)^{\alpha}$ $(\alpha \notin(-1 / 2,1 / 2))$ satisfies on every ellipse $\mathcal{E}_{\varrho}$ with $\varrho \geq \varrho_{*}$

$$
\max _{z \in \mathcal{E}_{e}}\left|K_{n+2}^{\mathrm{Lo}}\left(z ; w_{\alpha}\right)\right|= \begin{cases}\left|K_{n+2}^{\mathrm{Lo}}\left(z_{r} ; w_{\alpha}\right)\right| & \text { if }-1<\alpha \leq-1 / 2, n \geq 1, \\ \left|K_{n+2}^{\mathrm{Lo}}\left(z_{i} ; w_{\alpha}\right)\right| & \text { if } \alpha \geq 1 / 2, n \geq 3 .\end{cases}
$$

The parameter $\varrho_{*}$ is as in Theorem A if $\alpha \in(-1,-1 / 2]$, and as in Theorem 1.2 if $\alpha \geq 1 / 2$. 
Corollary 4.2. The kernel $K_{n+2}^{\mathrm{Lo}, d}\left(\cdot ; w_{\alpha}\right)$ of the $(n+2)$-point Gauss-Lobatto quadrature formula with double end nodes associated with the Gegenbauer weight function $w_{\alpha}(x)=\left(1-x^{2}\right)^{\alpha}(\alpha \in(-1,-1 / 2])$ satisfies on every ellipse $\mathcal{E}_{\varrho}$ with $\varrho \geq \varrho_{*}$

$$
\max _{z \in \mathcal{E}_{e}}\left|K_{n+2}^{\mathrm{Lo}, d}\left(z ; w_{\alpha}\right)\right|=\left|K_{n+2}^{\mathrm{Lo}, d}\left(z_{r} ; w_{\alpha}\right)\right| .
$$

The parameter $\varrho_{*}$ is as in Theorem 1.3 .

2. The conclusions of Corollaries 4.1 and 4.2 in the limit cases $\alpha= \pm 1 / 2$ can be sharpened. For the kernel of the Gauss-Lobatto quadrature formula associated with the first Chebyshev weight $w_{-1 / 2}(x)=\left(1-x^{2}\right)^{-1 / 2}$ Gautschi proved $([1$, Theorem 4.1]) that

$$
\max _{z \in \mathcal{E}_{\varrho}}\left|K_{n+2}^{\mathrm{Lo}}\left(z ; w_{-1 / 2}\right)\right|=K_{n+2}^{\mathrm{Lo}}\left(z_{r} ; w_{-1 / 2}\right) \quad \text { for all } \varrho>1 .
$$

For the kernel $K_{m+2}^{\mathrm{Lo}}\left(z ; w_{1 / 2}\right)$ Schira [9] proved that for $n \geq 3$

$$
\max _{z \in \mathcal{E}_{\varrho}}\left|K_{n+2}^{\mathrm{Lo}}\left(z ; w_{1 / 2}\right)\right|=\left|K_{n+2}^{\mathrm{Lo}}\left(z_{i} ; w_{1 / 2}\right)\right| \text { for all } \varrho>\varrho(n),
$$

where the parameter $\varrho(n)$ is expressed as the unique root of some nonlinear equation and satisfies

$$
\lim _{n \rightarrow \infty} \varrho(n)=1 .
$$

This confirms some empirical results about the behavior of $\left|K_{n+2}^{\mathrm{Lo}}\left(z ; w_{1 / 2}\right)\right|$ obtained in $[1]$.

For the kernel of the Gauss-Lobatto quadrature formula with double end nodes with respect to the first Chebyshev weight, Gautschi and $\mathrm{Li}$ [3] have proved that

$$
\max _{z \in \mathcal{E}_{e}}\left|K_{n+2}^{\mathrm{Lo}, d}\left(z ; w_{-1 / 2}\right)\right|=K_{n+2}^{\mathrm{Lo}, d}\left(z_{r} ; w_{-1 / 2}\right) \quad \text { for all } \varrho>1 .
$$

3. The restriction on $\varrho$ in Theorem 1.3 appears in order to ensure that Lemma 2.2 holds true. It was shown by Schira that, in a sense, the value $\varrho_{*}=\frac{\sqrt{2}}{2}(1+\sqrt{3})$ in Lemma 2.2 cannot be replaced by any smaller value.

However, an improvement is possible in Theorem 1.2. The values $\varrho_{*}=3.88$ and $\varrho_{*}=3.72$ are in fact the smallest values for which we have a proof that $\mu_{4}$ and $\mu_{5}$ attain maximal absolute value at $z=z_{i}$ provided $\varrho \geq \varrho_{*}$.

One could try to express the critical parameters $\varrho(m)$ ensuring that

$$
\max _{z \in \mathcal{E}_{\varrho}}\left|\mu_{m+1}(z)\right|=\left|\mu_{m+1}\left(z_{i}\right)\right| \text { for all } \varrho \geq \varrho(m)
$$

as solutions of certain equations, as was done in [9]. Alternatively, an upper bound for $\varrho(m)$ can be obtained by a direct application of Lemmas 2.3 and 2.4 to $\left|\mu_{m+1}(z)\right|$. However, both approaches seem to be quite laborious.

On the other hand, our method of proof implies that if (4.3) holds true for some fixed $m \geq 3$ with $\varrho(m) \geq 2.3$, then

$$
\max _{z \in \mathcal{E}_{\varrho}}\left|\mu_{m+2 j+1}(z)\right|=\left|\mu_{m+2 j+1}\left(z_{i}\right)\right| \text { for all } \varrho \geq \varrho(m), j=1,2, \ldots .
$$

Consequently, for $n \geq 3$ and weight functions $w$ satisfying the assumptions of Theorem 1.2 we have

$$
\max _{z \in \mathcal{E}_{\varrho}}\left|K_{n+2 j}^{\mathrm{Lo}}\left(z_{i} ; w\right)\right|=\left|K_{n+2 j}^{\mathrm{Lo}}\left(z_{i} ; w\right)\right| \quad \text { for all } \varrho \geq \varrho(n)(j=1,2, \ldots) .
$$

The same observation applies to the second case of Corollary 4.1. 
In Table 1 we present the numerical values of $\varrho(n)$ for $3 \leq n \leq 30$ and $n=60,100$.

TABLE 1. Values of $\varrho(n)$

\begin{tabular}{|c|c|c|c|c|c|c|c|c|c|c|}
\hline$n$ & 3 & 4 & 5 & 6 & 7 & 8 & 9 & 10 & 11 & 12 \\
$\varrho(n)$ & 3.8318 & 3.2308 & 3.0022 & 2.8791 & 2.8000 & 2.7447 & 2.7036 & 2.6718 & 2.6464 & 2.6256 \\
\hline$n$ & 13 & 14 & 15 & 16 & 17 & 18 & 19 & 20 & 21 & 22 \\
$\varrho(n)$ & 2.6083 & 2.5937 & 2.5811 & 2.5702 & 2.5607 & 2.5522 & 2.5447 & 2.5380 & 2.5319 & 2.5264 \\
\hline$n$ & 23 & 24 & 25 & 26 & 27 & 28 & 29 & 30 & 60 & 100 \\
$\varrho(n)$ & 2.5214 & 2.5168 & 2.5126 & 2.5087 & 2.5052 & 2.5019 & 2.4988 & 2.4959 & 2.4548 & 2.4385 \\
\hline
\end{tabular}

\section{REFERENCES}

[1] W. Gautschi, On the remainder term for analytic functions of Gauss-Lobatto and GaussRadau quadratures, Rocky Mountain J. Math. 21 (1991), 209-226. Corrected in W. Gautschi, Rocky Mountain J. Math. 21 (1991), 1143. MR 93a:41071a; MR 93a:41071b

[2] W. Gautschi, Remainder estimates for analytic functions, Numerical Integration (T. O. Espelid, A. Genz, eds.), Kluwer Academic Publishers, 1992, 133-145. MR 94e:41049

[3] W. Gautschi and S. Li, The remainder term for analytic functions of Gauss-Radau and Gauss-Lobatto quadrature rules with multiple end points, J. Comp. Appl. Math. 33 (1990), 315-329. MR 92a:65078

[4] W. Gautschi and R. S. Varga, Error bounds for Gaussian quadrature of analytic functions, SIAM J. Numer. Anal. 20 (1983), 1170-1186. MR 86j:65010

[5] W. Gautschi, E. Tychopoulos and R. S. Varga, A note on the contour representation of the remainder term for a Gauss-Chebyshev quadrature rule, SIAM J. Numer. Anal. 27 (1990), 219-224. MR 91d:65044

[6] D. B. Hunter, Some error expansions for Gaussian quadrature, BIT 35 (1995), 64-82. MR 97i: 65040

[7] F. Peherstorfer, On the remainder of Gaussian quadrature formulas for Bernstein-Szegö weight functions, Math. Comp. 60 (1993), 317-325. MR 93d:65030

[8] T. Schira, Ableitungsfreie Fehlerabschätzungen bei numerischer Integration holomorpher Funktionen, Ph.D. Dissertation, Univ. Karlsruhe, 1994.

[9] T. Schira, The remainder term for analytic functions of Gauss-Lobatto quadratures, J. Comp. Appl. Math. 76 (1996), 171-193. MR 97m:41033

[10] T. Schira, The remainder term for analytic functions of symmetric Gaussian quadratures, Math. Comp. 66 (1997), 297-310. MR 97c:65050

Department of Computing and Mathematics, University of Bradford, BD7 Bradford, West Yorkshire, United Kingdom

Department of Mathematics, University of Sofia, Blvd. James Bourchier 5, 1164 Sofia, Bulgaria

E-mail address: geno@fmi.uni-sofia.bg 